\title{
Characteristic Research of $\mathrm{La}_{3} \mathrm{Ga}_{5} \mathrm{SiO}_{14}$ in Q-switched Laser
}

\author{
Yan Sun \\ Aviation University of Air Force,Changchun 130022,China \\ 1309530560@qq.com
}

Keywords: LGS, Pockels' cell, Electro-optical Q-switch

Abstract. In this thesis, the related parameters of LGS are treated in analysis. We use experiment method to investigate LGS's characteristics as Q-switched crystal in laser and compare it with the commonly used KD*P crystal. The advantages and disadvantages of LGS crystal are summarized in the photoelectric effect application.

\section{LGS's Characteristics}

After years of research, theoretical and experimental research in tuning Q laser and laser material has made great development [1-5]. LGS $\left(\mathrm{La}_{3} \mathrm{Ga}_{5} \mathrm{SiO}_{14}\right)$ is an excellent nonlinear crystal. The crystal structure of this type is shown in Fig 1 [6].LGS has a very distinct electro-optical effect, the reason why it can be used as an electro-optical Q-switched crystal.

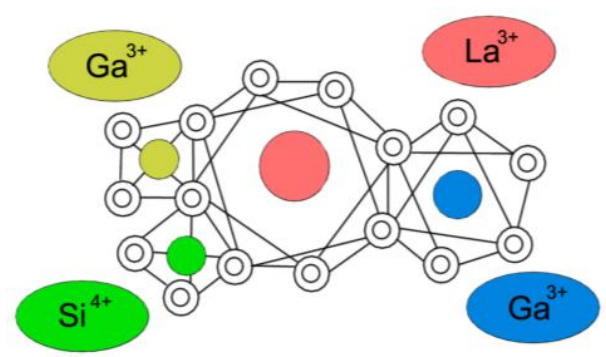

Fig.1 LGS crystal structure

The main physical and chemical properties of LGS:

\begin{tabular}{ll}
\hline Density & $5.754 \mathrm{~g} / \mathrm{cm}^{2}$ \\
\hline Melting point & $470^{\circ} \mathrm{C}($ Transformation point: N/A $)$ \\
\hline Transmittance wavelength & $242-3200 \mathrm{~nm}$ \\
\hline Specific heat & $0.47 \mathrm{~J} / \mathrm{gm}-\mathrm{K}$ at $300 \mathrm{~K}$ \\
\hline Refractive index parameter & $\mathrm{Nz}=1.89$ \\
\hline Electro-optic coefficient & $\mathrm{R} 41=1.8 \mathrm{pm} / \mathrm{v} \mathrm{r} 11=2.3 \mathrm{pm} / \mathrm{v}$ \\
\hline Deliquescence & No deliquescence \\
\hline $\begin{array}{l}\text { Thermal } \\
\text { coefficient }\end{array}$ & $\mathrm{a}_{11}=5.15 \mathrm{E}-6 \mathrm{~K}^{\prime}(\perp \mathrm{Z}) \mathrm{a}_{33}=3.65 \mathrm{E}-6 \mathrm{~K}^{`}(/ / \mathrm{Z})$ \\
\hline
\end{tabular}

The main parameters of LGS as Q-switch crystal:

\begin{tabular}{ll}
\hline $\mathrm{V}_{\lambda / 4}$ & $17000 *(\mathrm{~d} / \mathrm{l}) \mathrm{V} @ 1064 \mathrm{~nm}$ \\
\hline Insertion loss & $<2 \%$ \\
\hline Extinction ratio & $>1000: 1$ \\
\hline Coating & $@ 1064 \mathrm{~nm}$ \\
\hline Wavefront aberration & $<\lambda / 4 @ 1064 \mathrm{~nm}$ \\
\hline Optical damage threshold & $900 \mathrm{MW} / \mathrm{cm}^{2}$ \\
\hline
\end{tabular}


By analyzing the features we know LGS is not deliquescent,so it can be directly exposed to the air when installation. Also because of this, LGS may be plated with antireflection coating at both ends, that greatly increasing its transmission rate, so that it can reach more than $98 \%$.In addition, if the crystal face is once accidentally damaged in use, LGS can be polished and re-coating to restore some losses. LGS is without phase change between room temperature and its melting point, so in theory LGS has excellent temperature stability, and is not like KD*P fogged at higher temperature.

\section{Experimental Apparatus}

Laser Device. LGS is installed in the laser as electro-optical Q-switched crystal. The laser structure is shown in Fig.2. The Laser consists of total reflection mirror(1), partial detector (2), Pockels' cell(3), polarizer(4), Xenon lamp(5), Nd:YAG(6), pump cavity(7) and output mirror(8).

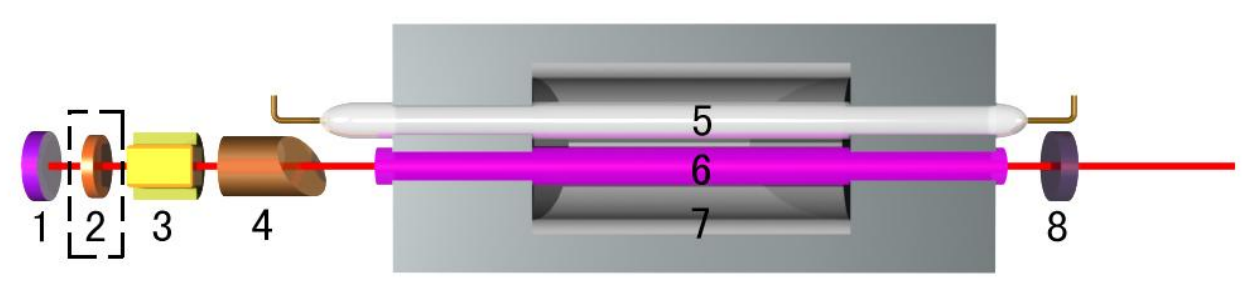

Fig.2 Electro-optical Q-switched Nd:YAG solid state laser structure

LGS Crystal Pockels' Cell. Two pieces of LGS crystal, used in the experiment are provided by Institute of Crystal Materials Shandong University. Their specifications are $8 \times 8 \times 38(\mathrm{~mm})$ and $10 \times 10 \times 48(\mathrm{~mm})$. In order to compare the different characteristics of crystals of different sizes, we ordered two sets of Pockels electro-optic cells. In addition to the size difference between the two sets of device, the structures are exactly the same. The concrete structure is shown in Fig. 3. LGS not deliquescence, so in the open air the crystal can be encapsulated in resin material Pockels' cell. In addition, the processing of resin shell should be done to ensure the tolerance and surface gloss, so that we can effectively improve the accuracy of the experiment, to avoid damage of crystal.

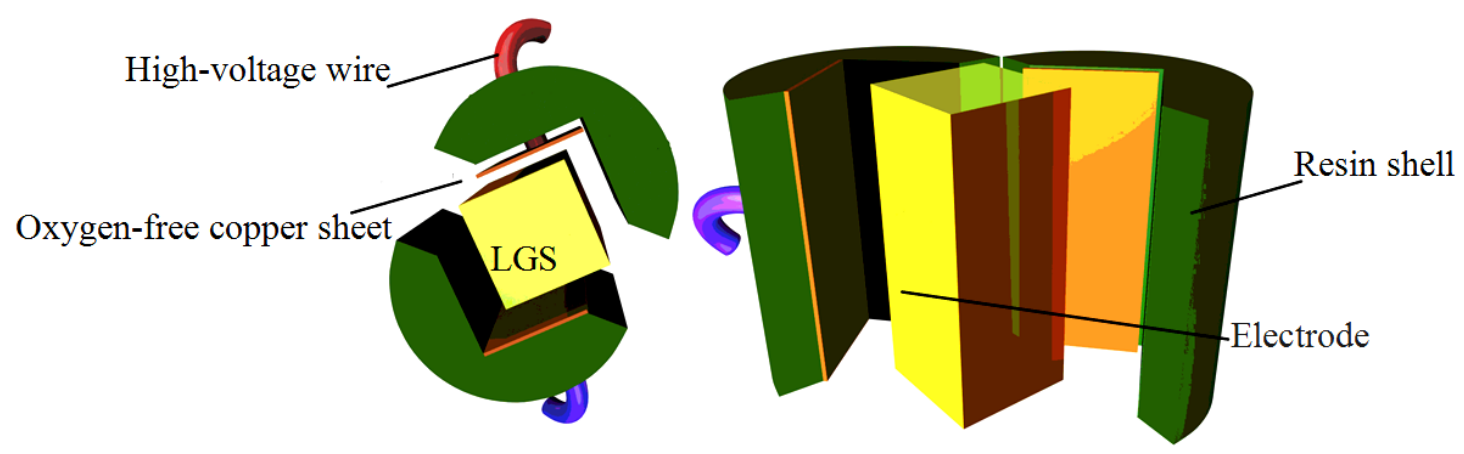

Fig.3 LGS Pockels' cell structure

In the packaging, we need to polish and level oxygen free copper sheets. Then the wires are welded to the backs of the oxygen-free coppers. We flat the coppers on the inner surface of shell and make the wire through the shell. When two pieces of copper welding have been completed, place LGS crystal in a separate half of shell, then lightly fasten the other half shell, carefully secure. 


\section{Experimental Study}

LGS Q-switched Laser Output Pulse Waveform. LGS Q-switched static light pulse is shown in Fig.4, and its dynamic laser pulse is shown in Fig.5.

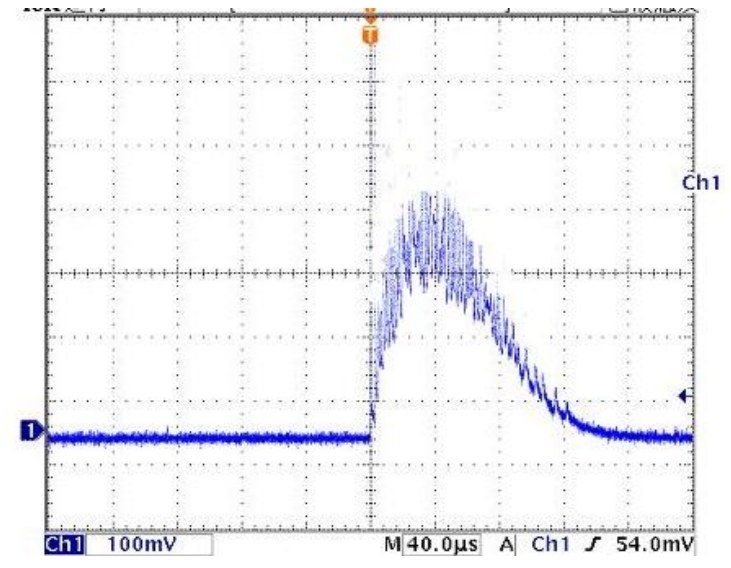

Fig.4 LGS Q-switched static laser pulse

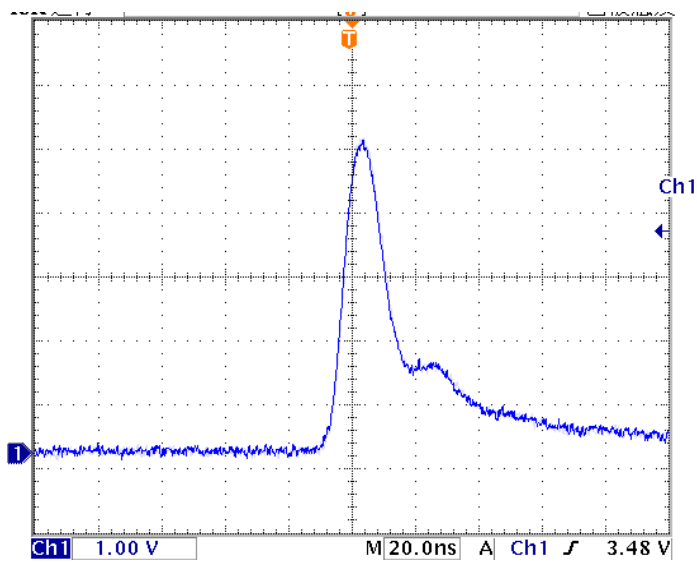

Fig.5 LGS Q-switched dynamic laser pulse

Dynamic Laser Pulse Width. Dynamic laser pulse width is shown in Tab.1. From the experiments, we can see that LGS is better than KD*P according to the obtained width data with the present experimental apparatus. In terms of LGS adding voltage Q-switch the pulse has been compressed to less than $15 \mathrm{~ns}$. Compared with the previous results done, it has reached a good level. LGS adding voltage Q-switch has many advantages of a high response speed, small laser jitter, strong security and high stability. So LGS for adding voltage Q-switch should be given affirmation. Previously, few people used a LGS for back voltage Q-switch, this time we pay attention to and focus on the back voltage Q-switch experiment. LGS experiment pulse width has been compressed to a considerable degree of KD*P pulse, 17.4ns. LGS also has shown potential as a back-pressure Q-switching. In this case, we have to consider the LGS's advantages of no deliquescence, high optical damage threshold and continuous operation in high temperature environments. Thus under various conditions and environmental experiments, LGS's unique advantage is more obvious as electro-optic Q-switching.

Tab.1 Electro-optical Q-switched dynamic laser pulse width

\begin{tabular}{|c|c|c|}
\hline & $\begin{array}{c}\text { Back voltage } \\
\text { laser pulse width(ns) }\end{array}$ & $\begin{array}{c}\text { Adding voltage } \\
\text { laser pulse width(ns) }\end{array}$ \\
\hline KD ${ }^{*} \mathrm{P}$ & 18.9 & 19.5 \\
\hline LGS & 17.7 & 14.7 \\
\hline
\end{tabular}

Tab.2 640V Operating voltage

\begin{tabular}{|l|c|c|c|}
\hline & LGS $8 \times 8 \times 38(\mathrm{~mm})$ & LGS $10 \times 10 \times 48(\mathrm{~mm})$ & KD*P \\
\hline Dynamic energy $(\mathrm{mJ})$ & 216 & 294 & 206 \\
\hline Static energy $(\mathrm{mJ})$ & 238 & 328 & 245 \\
\hline Dynamic to static ratio & 0.91 & 0.90 & 0.84 \\
\hline
\end{tabular}

Dynamic to Static Ratio. Dynamic to static ratio is shown in Tab.2. Dynamic to static ratio represents Q-switch efficiency, because the insertion loss of electro-optic crystal itself and the rotation angle will influence the efficiency. The experimental data show that for two sizes of 
Q-switch LGS, the measured Dynamic to static ratio is greater than KD*P crystals. Seen from Q-switch efficiency point of view, LGS as a Q-switching is more appropriate.

\section{Summary}

The related characteristics of LGS crystal are summarized into the following:

LGS's Advantages. One advantage is high transmittance. In Experiment, LGS's convenient use and its high transmittance are very conspicuous. This is a considerable part of the reason for this benefit from double-sided antireflective coating. A direct result of the high transmittance is to reduce the insertion loss and improve the movement ratio, thereby improve the overall efficiency of the laser.

Another advantage is strong anti optical damage. Theoretically, LGS anti-damage threshold is 900MW $/ \mathrm{cm}^{2}$, nine times higher than LN crystal.

LGS's Disadvantages. One disadvantage is with not enough high extinction ratio. In experiments, we found that after the power supply voltage is higher than $700 \mathrm{~V}$, no matter how to improve crystal high voltage, Q switch can not be completely. This should be caused by the experimental crystal with not high extinction ratio. As a new type of artificial crystal, LGS's such defects may be the result of imperfect growth process. With improvements in the production process, the defect should be expected to be overcome.

Another disadvantage is incomplete process, some unreasonable doping concentration. As we mentioned earlier, that LGS extinction ratio is not high may be caused by the growth of immature technology. As a new type of artificial crystal developed in recent years, its doping is appropriate, the concentration should control how much, these are problems to be solved. Meanwhile, too long crystal growth cycle is also an important issue.

\section{References}

[1] J.J.Degnan.IEEE J. Quant. Electron. 25(2):214-220(1989)

[2] Xingyu Zhang,Shengzhi Zhao,Qingpu Wang et al. . IEE E. J .Quant. Electron. 35(12): 1912-1918(1999)

[3] J. J. Zayhow ski, C. Dill III. Opt.Lett. 17(17):1201-1203(1992).

[4] J. J. Zayhow ski, P. L. Kelley.IEEE J.Quant. Electron . 27(9):2220-2225(1991)

[5] W.Koechner. Springer Verlag .Ch8(1999)

[6] Jiyang Wang,Rongjiang Han,Xin Yin et al. In Chinese. Functional Materials.34(1):9-12(2003) 